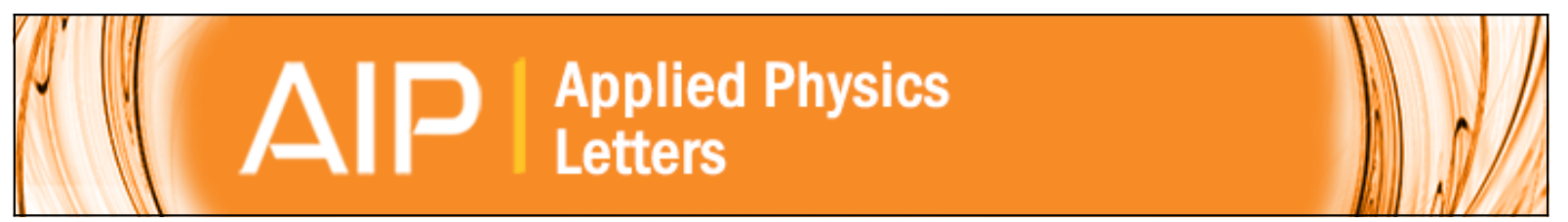

\title{
Impact of lateral carrier confinement on electro-optical tuning properties of polariton condensates
}

S. Brodbeck, H. Suchomel, M. Amthor, A. Wolf, M. Kamp, C. Schneider, and S. Höfling

Citation: Applied Physics Letters 107, 041108 (2015); doi: 10.1063/1.4927601

View online: http://dx.doi.org/10.1063/1.4927601

View Table of Contents: http://scitation.aip.org/content/aip/journal/apl/107/4?ver=pdfcov

Published by the AIP Publishing

\section{Articles you may be interested in}

Electro optical tuning of Tamm-plasmon exciton-polaritons

Appl. Phys. Lett. 105, 181107 (2014); 10.1063/1.4901023

On Bose condensation of excitons in quasi-two-dimensional semiconductor heterostructures Low Temp. Phys. 38, 541 (2012); 10.1063/1.4733681

Polariton parametric luminescence in a single micropillar

Appl. Phys. Lett. 90, 051107 (2007); 10.1063/1.2435515

Tunnel-assisted manipulation of intersubband polaritons in asymmetric coupled quantum wells

Appl. Phys. Lett. 89, 171109 (2006); 10.1063/1.2367664

Enhancement of the quantum-confined stark effect utilizing asymmetric quantum well structures Appl. Phys. Lett. 74, 254 (1999); 10.1063/1.123272






\title{
Impact of lateral carrier confinement on electro-optical tuning properties of polariton condensates
}

\author{
S. Brodbeck, ${ }^{1}$ H. Suchomel, ${ }^{1}$ M. Amthor, ${ }^{1}$ A. Wolf, ${ }^{1}$ M. Kamp,,${ }^{1}$ C. Schneider, ${ }^{1,2}$ \\ and S. Höfling ${ }^{1,3}$ \\ ${ }^{1}$ Technische Physik, Physikalisches Institut and Wilhelm Conrad Röntgen-Research Center for Complex \\ Material Systems, Universität Würzburg, Am Hubland, D-97074 Würzburg, Germany \\ ${ }^{2}$ Mediterranean Institute of Fundamental Physics, Via Appia Nuova 31, 00040 Rome, Italy \\ ${ }^{3}$ SUPA, School of Physics and Astronomy, University of St. Andrews, St. Andrews KY 16 9SS, United Kingdom
}

(Received 23 June 2015; accepted 18 July 2015; published online 28 July 2015)

\begin{abstract}
Electro-optical measurements on exciton-polaritons below and above the condensation threshold are performed on high quality, pin-doped microcavities with embedded GaAs quantum wells. Applying an external electric field shifts the polariton emission by hundreds of $\mu \mathrm{eV}$ both in the linear and the nonlinear regime. We study three device geometries to investigate the influence of carrier confinement in the plane of the quantum well on the electro-optical tuning properties. In the conventional micropillar geometry, the electric field tuning behavior is dominated by the effects of carrier tunneling and electric field screening that manifest in a blueshift of the polariton emission. In stark contrast, for a planar sample geometry, we can significantly extend the range of electric fields and a redshift is observed. To separate the contributions of quantum confined Stark effect and reduced exciton oscillator strength to the energy shift, we study a third sample where the etching of micropillars is stopped just above the active region. In this semi-planar geometry, exciton and polariton emissions can be measured simultaneously. As for the planar geometry, redshifts of the polariton emission are observed below and above threshold that are well reproduced by theoretical shifts. (C) 2015 AIP Publishing LLC. [http://dx.doi.org/10.1063/1.4927601]
\end{abstract}

Semiconductor quantum well microcavities have emerged as an attractive system to study the condensation of bosonic particles at elevated temperatures, namely, of exciton-polaritons. ${ }^{1,2}$ These are mixed light-matter quasiparticles arising from the strong coupling of quantum well excitons and cavity photons. ${ }^{3}$ Due to their photonic component, they exhibit a very light effective mass ${ }^{4}$ and are easily accessible in luminescence measurements. Polariton condensation can be observed at temperatures up to and exceeding $300 \mathrm{~K}$ in semiconductors with large exciton binding energies such as $\mathrm{GaN},{ }^{5} \mathrm{ZnO},{ }^{6}$ and organic semiconductors, ${ }^{7-9}$ which highlights the potential for devices based on polaritoncondensation such as lasers without the need for population inversion. $^{10,11}$

Due to their excitonic component, polaritons interact strongly with external fields and the carrier reservoir. These strong interactions can be conveniently exploited in polaritonic integrated architectures ${ }^{12}$ where the polariton flow can be directed and manipulated by external lasers, ${ }^{13-15}$ but, in principle, also by simple electric gates. In an electric field along the growth direction, the polariton emission exhibits a redshift due to the Stark shift of the exciton ${ }^{16}$ which has been observed in the linear regime below the condensation threshold. ${ }^{17}$ Furthermore, the exciton oscillator strength decreases due to decreasing overlap of electron and hole wavefunctions which reduces the Rabi splitting. ${ }^{18}$ This causes a blueshift of the polariton emission, but usually the redshift dominates. In a recent study ${ }^{19}$ of the polariton emission below and above condensation threshold, surprisingly, a significant blueshift was observed with increasing electric field. This was explained by longitudinal optical
(LO)-phonon assisted tunneling which leads to carrier accumulation in one quantum well of the incorporated quantum well stacks. This quantum well is spectrally detuned from the remaining quantum wells and consequently the Rabi splitting is reduced. Here, we show that this blueshift can be suppressed by choosing a planar or quasi-planar sample geometry, and we restore the conventional quantum confined Stark effect of the polariton condensate which could be used to control polariton-transistors ${ }^{20}$ or to electrostatically define trapping potentials for polaritons.

We study three different sample geometries: micropillar, planar, and semi-planar. The quantum wells are left unetched for the latter two geometries. Samples were grown by molecular beam epitaxy on n-doped (001) GaAs substrates. They consist of a $\lambda / 2$-AlAs cavity surrounded by AlAs/ $\mathrm{Al}_{0.2} \mathrm{Ga}_{0.8} \mathrm{As}$ distributed Bragg reflectors (DBRs) with 23.5 (27) mirror pairs in the top (bottom) reflector. A single stack of $4 \mathrm{GaAs}$ quantum wells with $7 \mathrm{~nm}$ width separated by $4 \mathrm{~nm}$ AlAs barriers is placed in the center of the cavity which has a nominal length of $l_{C}=100 \mathrm{~nm}$. The AlAs barriers inhibit tunneling of carriers out of the quantum well stack up to very large electric fields. Quasi-graded superlattices ${ }^{21}$ were introduced at the heterointerfaces of both DBRs and at the cavity-DBR interfaces. Replacing the abrupt AlAs-AlGaAs interfaces with $20 \mathrm{~nm}$ wide superlattices lowers the series resistance of the DBRs. The top (bottom) DBR is p-doped with carbon (n-doped with silicon). Doping concentrations were reduced from $3 \times 10^{18} \mathrm{~cm}^{-3}$ to $1 \times 10^{18} \mathrm{~cm}^{-3}$ towards the intrinsic cavity. ${ }^{10}$ The topmost mirror pair of the upper DBR is doped at a concentration of $2 \times 10^{19} \mathrm{~cm}^{-3}$ to ensure a low contact resistance. The Rabi splitting amounts to $(8.7 \pm 0.1) \mathrm{meV}$ 
as determined from low temperature white light reflectance measurements where the cavity resonance was tuned through the exciton energy by varying the radial position of the light spot over the unprocessed wafer. In this experiment, we intentionally reduced the reflectivity of the top DBR by removing 13 mirror pairs from the top DBR via dry etching to ensure a sufficient signal to noise ratio.

The micropillar and planar samples are fabricated on different pieces of the same wafer. Micropillars are processed similarly to the sample studied in Ref. 10. The ncontact is formed by evaporating an AuGe-Ni-Au alloy on the backside of the substrate. Next, $20 \mu$ m wide circular pillars are defined by optical lithography and electron cyclotron resonance reactive ion etching with an $\mathrm{Ar} / \mathrm{Cl}$ plasma. The etching is stopped in the substrate. The sample is then planarized with a polymer (benzocyclobutene) and ring shaped p-contacts (Au-Ti-alloy) are deposited on top of the pillars. For the planar geometry, we use a similar process to the one used in Ref. 17. After deposition of the n-contact on the backside of the substrate, metal discs with a diameter of $40 \mu \mathrm{m}$ are defined by electron beam lithography and evaporation of a $40 \mathrm{~nm}$ thick gold layer. The semi-planar sample is fabricated from a different wafer where only the first inner 4 mirror pairs of the upper DBR are doped $\left(2\right.$ at $2 \times 10^{18} \mathrm{~cm}^{-3}$ and 2 at $1 \times 10^{19} \mathrm{~cm}^{-3}$ ). Micropillars with $20 \mu \mathrm{m}$ diameter are again defined by optical lithography and dry etching, but the etching is stopped in the upper DBR when the highly doped mirror pairs are reached. Ring shaped p-contacts with an inner diameter of $26 \mu \mathrm{m}$ are then deposited around the pillar onto the p-doped layers of the upper DBR. As for the other samples, the n-contact is deposited on the backside of the substrate.

Angle-resolved electro-optical measurements are performed in a Fourier-space micro-photoluminescence (PL) setup. ${ }^{2,22}$ The samples are mounted in a helium flow cryostat with electrical feedthroughs and kept at $5 \mathrm{~K}$ during the measurements. They are excited at normal incidence by a Ti:sapphire laser with a spot size of $40 \mu \mathrm{m}$ that provides $50 \mathrm{ps}$ long laser pulses with a repetition rate of $82 \mathrm{MHz}$. The laser wavelength is tuned to the first reflectance minimum outside the stopband $95 \mathrm{meV}$ above the lower polariton energy. A voltage source is connected to the top and bottom contacts of the samples to vary the electric field $F$ during measurements. The nominal electric field across the cavity is calculated as $F=\left(U_{0}-U\right) / l_{c}$, where $U_{0}$ is the knee voltage and $U$ is the external voltage. We note that the actual electric field strength across the quantum wells may be significantly lower than the nominal field strength due to screening effects.

In Fig. 1, we show electro-optical tuning experiments for the micropillar sample. A photon-exciton detuning of $-8.2 \mathrm{meV}$ without externally applied voltage (open circuit configuration) was chosen for this measurement. Analyzing the emission at $k_{\|}=0$ with increasing pump power when the voltage source is not connected, Fig. 1(c), yields the typical signatures of polariton condensation at a threshold power of $(6.5 \pm 0.5) \mathrm{mW}$, namely, a non-linear increase of the groundstate emission at the threshold pumping power accompanied by linewidth narrowing and a blueshift of the emission due to repulsive polariton-polariton and polariton-reservoir interactions. ${ }^{23}$ Figs. 1(a) and 1(b) show momentum resolved PL spectra below and above the condensation threshold with the left half of each spectrum recorded at $F=0 \mathrm{kV} / \mathrm{cm}$ and the right half recorded at $F=162 \mathrm{kV} / \mathrm{cm}$. A clear blueshift with increasing electric field is observed as reported previously. ${ }^{19}$ For a more detailed analysis of the emission shifts, we extract line-spectra at $k_{\|}=0$ from the momentum resolved spectra recorded at different electric fields. Line-spectra are shown in Fig. 1(d) and the extracted emission energies below and above threshold as a function of electric field are plotted in Fig. 1(e). The observed shift of the emission is very similar at both excitation powers supporting that strong lightmatter coupling is preserved above the non-linearity threshold. For $30 \mathrm{kV} / \mathrm{cm}<F<300 \mathrm{kV} / \mathrm{cm}$, continuous shifts of up to $+0.48 \mathrm{meV}$ below threshold and $+0.40 \mathrm{meV}$ above threshold are observed. We believe that this blueshift can be explained by carrier tunneling between the quantum wells as proposed in Ref. 19. At $F=30 \mathrm{kV} / \mathrm{cm}$, the lowest energy levels in the conduction band of neighboring quantum wells are separated by the energy of one LO-phonon of $36 \mathrm{meV}$ (Ref. 24) as we calculated by numerically solving the Poisson- and Schrödinger-equations for our device.
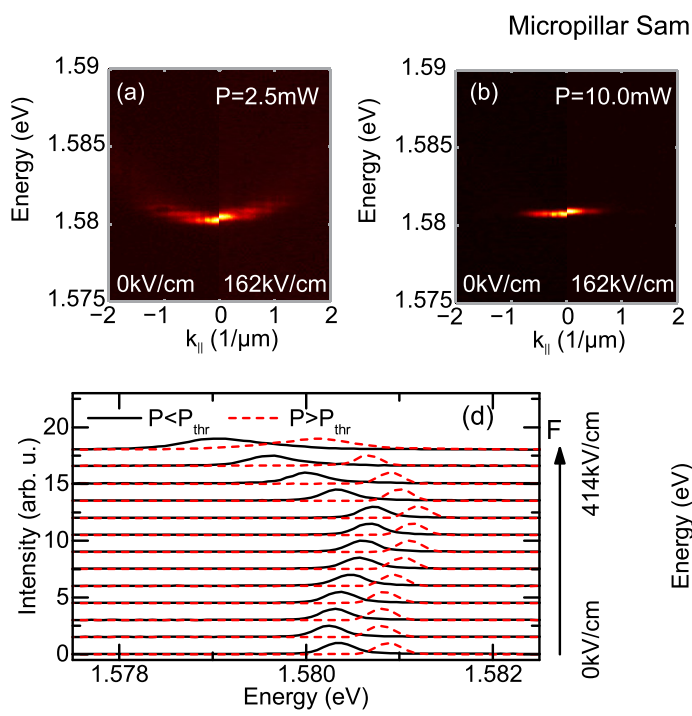
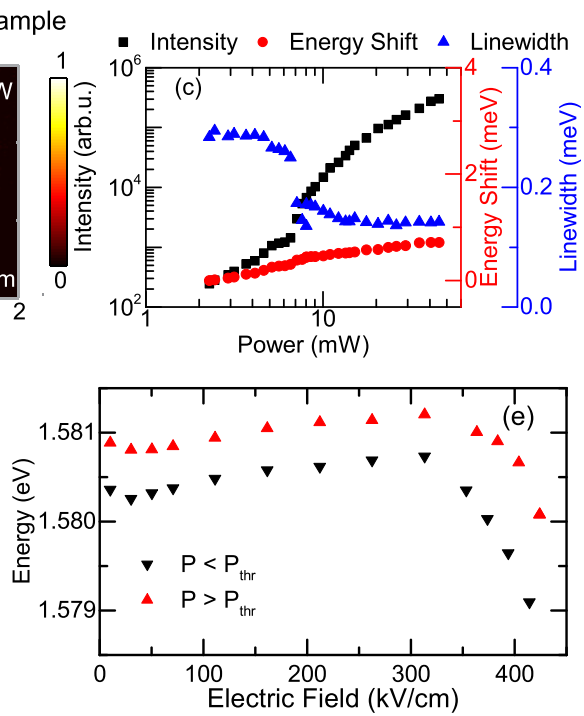

FIG. 1. Electro-optical tuning measurement on the micropillar sample. (a) Momentum resolved PL spectra below condensation threshold at a nominal electric field $F=0 \mathrm{kV} / \mathrm{cm}$ (left half) and at $F=162 \mathrm{kV} / \mathrm{cm}$. (b) Same as (a) above the polariton condensation threshold. (c) Power dependence of ground state emission intensity, energy, and linewidth measured without an externally applied voltage. (d) Line-spectra at $k_{\|}=0$ extracted from momentum resolved PL spectra for increasing electric field. (e) Fitted peak energies of the spectra shown in (d) as a function of electric field. 

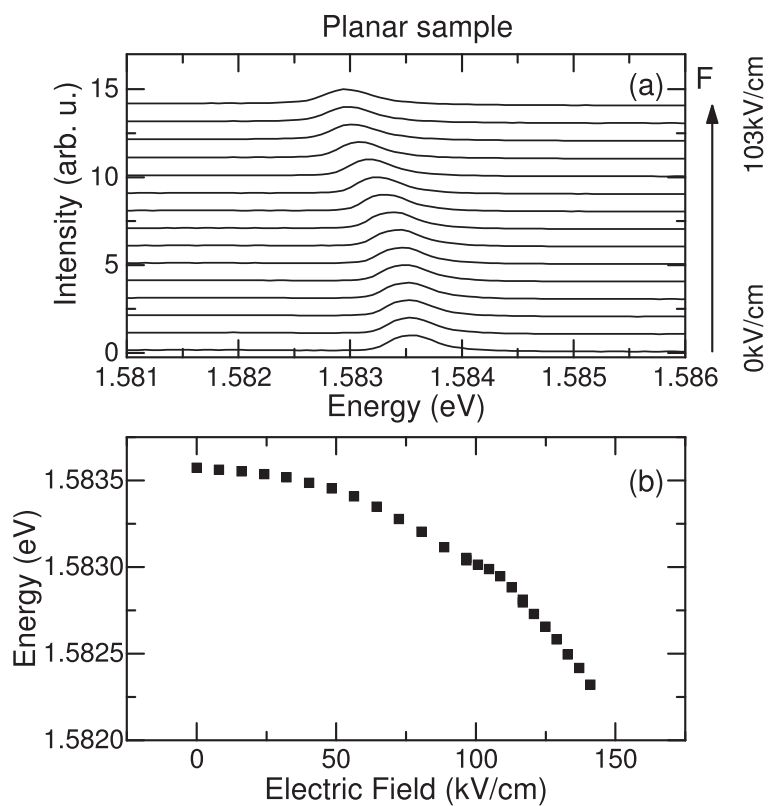

FIG. 2. Electro-optical tuning of the planar sample at an excitation power $p=3 \mathrm{~mW}$. Note that this sample and the micropillar sample were fabricated from the same wafer. (a) Line-spectra at $k_{\|}=0$ extracted from momentum resolved PL spectra for increasing electric field. (b) Polariton emission energy as a function of electric field.

Therefore, electrons accumulate in the bottom quantum well due to LO-phonon-assisted tunneling. They screen the external electric field and lift the degeneracy of exciton energies in the quantum well stack. The effective number of quantum wells participating in the strong light-matter coupling is reduced to three and the Rabi splitting is reduced by a factor of $\sqrt{3 / 4}$ (Ref. 25) to $7.5 \mathrm{meV}$. This results in an increase of the lower polariton energy by $0.4 \mathrm{meV}$ at the selected detuning which is in good agreement with the experimentally observed values. As the exciton linewidth in the bottom quantum well increases drastically due to the additional carriers ${ }^{19}$ no additional polariton branch appears despite the split exciton energies. For large electric fields, a strong redshift is observed accompanied by linewidth broadening and reduction of the emission intensity. In this last regime, the bands may be tilted sufficiently strong to allow carriers to tunnel out of the quantum well stack. This would reduce the screening of the external electric field and thus the previous blueshift of the emission.

Next, we consider the planar sample. For this device, a detuning of $-4.0 \mathrm{meV}$ is determined in the open circuit configuration. Line-spectra at $k_{\|}=0$ are shown in Fig. 2(a) at low excitation power for increasing electric field, and the emission energies extracted from the spectra are shown in Fig. 2(b). In stark contrast to the measurement on the micropillar sample presented in Fig. 1, here the polariton energy is redshifted with increasing electric field by up to $-1.2 \mathrm{meV}$ at $140 \mathrm{kV} / \mathrm{cm}$. As both samples were fabricated from the same wafer and are showing almost identical detuning, this different tuning behavior can only be explained by the different sample geometries. In the micropillar sample, carriers accumulating in one quantum well due to tunneling are confined to the etched structure of the pillar with a radius that is on the order of diffusion lengths in intrinsic GaAs of tens of $\mu \mathrm{m} .{ }^{26}$ In the planar sample on the other hand, electrons

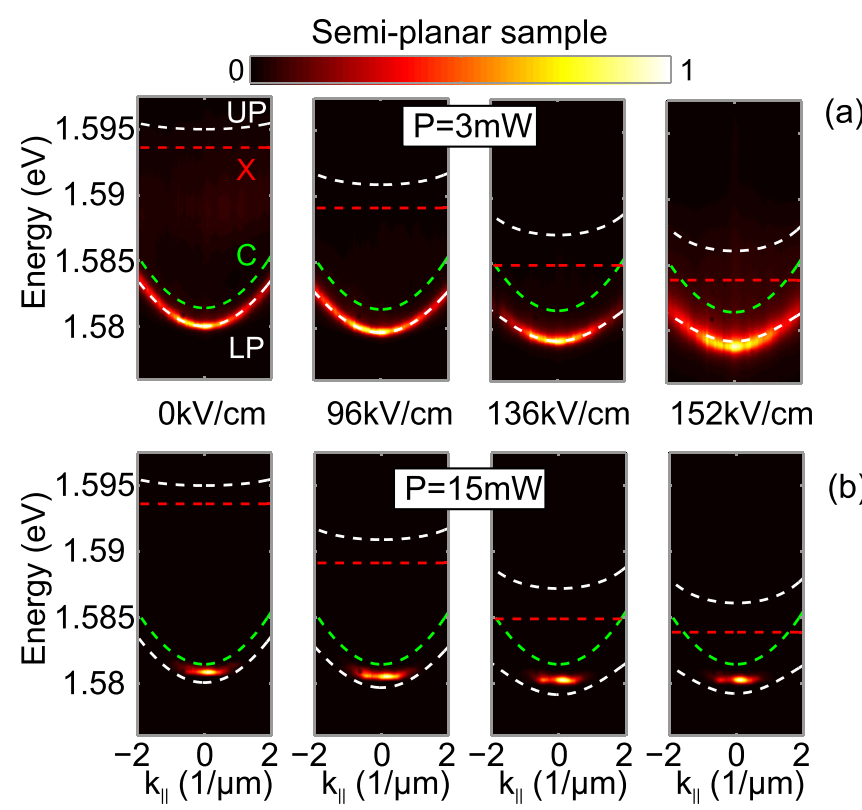

FIG. 3. Electro-optical tuning measurement on the semi-planar sample. (a) PL spectra below condensation threshold at various electric fields. White dashed lines are polariton dispersions resulting from a coupled harmonic oscillator fit. Red and green dashed lines are dispersions of bare exciton and cavity photon, respectively. (b) Same as (a) above condensation threshold.

spread out in the plane of the quantum well due to diffusion and Coulomb repulsion. Thus, the carrier density required for screening effects to become noticeable is reached at considerably larger nominal electric fields. At low excitation powers, the photoluminescence is quenched for $F>$ $150 \mathrm{kV} / \mathrm{cm}$ due to tunneling losses, and therefore no blueshift is observed.

To separate the previously mentioned contributions of quantum confined Stark effect and reduced exciton oscillator strength to the energy shift of polaritons, we investigate the semi-planar sample. For this sample, emission of the uncoupled exciton can be collected at low excitation powers from the surrounding of the pillar which remains in the weak coupling regime due to the DBR removal. Figs. 3(a) and 3(b) show momentum resolved PL spectra below and above the polariton condensation threshold for various electric fields. The detuning and threshold power determined in open circuit configuration amount to $-7.6 \mathrm{meV}$ and $(6.1 \pm 0.5) \mathrm{mW}$, respectively. As for the planar sample, the polariton emission redshifts with increasing field strength by up to $-1.1 \mathrm{meV}$ $(-0.58 \mathrm{meV})$ at $150 \mathrm{kV} / \mathrm{cm}$ below (above) threshold. The lower polariton dispersions measured below threshold are fit with a coupled oscillator model where the Rabi splitting is the only fitting parameter. The exciton energy is directly measured by collecting emission from the surrounding of the pillar, while the cavity energy is determined from a fit to the PL spectrum recorded in the open circuit configuration and then kept constant for the remaining fits. Analysis of the line-spectra at $k_{\|}=0$, Fig. 4(a), yields the polariton emission energies as a function of electric field, Fig. 4(b). We observe a continuous shift to lower energies both below and above condensation threshold, again supporting that the system is still in the regime of strong coupling above the non-linearity threshold. For large electric fields, the redshift of the condensate emission is partially reversed before the condensate 



FIG. 4. (a) PL spectra at $k_{\|}=0$ measured on the semi-planar sample below and above condensation threshold for increasing electric field. The emission below threshold is quenched for $F>150 \mathrm{kV} / \mathrm{cm}$ due to the large losses caused by carrier tunneling. (b) Fitted peak energies of the bias-dependent PL measurements. The calculated shifts due to exciton Stark shift and reduced Rabi splitting are shown as dashed lines. (c) Measured exciton energy (left axis) and Rabi splitting determined by fitting momentum resolved spectra at low excitation powers (right axis) as a function of electric field. Dashed lines are calculations based on theoretical polarizability (exciton energy) and on the spatial overlap of electron and hole wave functions (Rabi splitting).

breaks up. This is only observed for excitation powers well above the condensation threshold. We attribute this behavior to screening effects as discussed for the micropillar sample, but due to the lack of in-plane carrier confinement large electric fields and pumping powers are necessary to observe them in the semi-planar sample.

The measured exciton energy and the Rabi splitting deduced from the coupled oscillator fits are plotted in Fig. 4(c). Both curves show the expected behavior of decreasing energy ${ }^{16}$ and splitting ${ }^{18}$ with increasing field, and they are in good agreement with the calculated values up to an electric field of $120 \mathrm{kV} / \mathrm{cm}$. For the exciton, we calculate the shift $\Delta E=-\alpha|F|^{2}$ according to Ref. 27. The calculation for a $7 \mathrm{~nm}$ wide GaAs quantum well in AlAs barriers yields a polarizability of $\alpha=5.61 \times 10^{-4} \mathrm{meV} \mathrm{kV}^{-2} \mathrm{~cm}^{2}$. For the Rabi splitting, first, the spatial overlap $O_{e h}$ of onedimensional electron and hole wave functions $f_{e}$ and $f_{h}$ along the growth direction was calculated numerically for a single quantum well at various electric fields as $O_{e h}=\left|\int f_{e} f_{h} d x\right|$. Then, the Rabi splitting $\Omega(F)$ normalized to the Rabi splitting at $F=0 \mathrm{kV} / \mathrm{cm}$ was calculated as $O_{e h}(F) / O_{e h .0}$, with $O_{e h}^{2}$ being proportional to the exciton oscillator strength. ${ }^{25}$ Dashed lines in Fig. 4(b) show the predicted polariton energies based on the calculated exciton energy and Rabi splitting. The theoretical curves are again in good agreement with the experimental values up to $F=120 \mathrm{kV} / \mathrm{cm}$.

To conclude, we have investigated the polariton emission both below and above condensation threshold in an electric field perpendicular to the quantum wells. An initial redshift of the emission is caused by the exciton Stark shift and a reduction of the exciton oscillator strength. A reversal of the redshift and subsequent blueshift of the polariton emission is observed at larger electric fields when LO-phonon-assisted tunneling between the quantum wells leads to screening of the external electric field. The onset of screening can be delayed by choosing a planar sample geometry. Our results show a pathway towards electrostatic trapping and manipulation of polaritons via the DC stark effect. More importantly, our scheme can be adapted in sophisticated polariton channel structures and interferometers to control and direct the polariton flow in integrated circuit schemes. ${ }^{15,28,29}$ In combination with electrical polariton injection ${ }^{10}$ and electrical polariton read out, ${ }^{30}$ this could pave the way towards ultra compact, all electrical polariton integrated logic circuits.

We thank M. Wagenbrenner and M. Emmerling for assistance during sample growth and fabrication and $\mathrm{K}$. Winkler for assistance during measurements. This work was supported by the State of Bavaria. S.H. gratefully acknowledges support by the Royal Society and the Wolfson Foundation.

${ }^{1}$ H. Deng, G. Weihs, C. Santori, J. Bloch, and Y. Yamamoto, Science 298, 199 (2002).

${ }^{2}$ J. Kasprzak, M. Richard, S. Kundermann, A. Baas, P. Jeambrun, J. M. J. Keeling, F. M. Marchetti, M. H. Szymanska, R. André, J. L. Staehli, V. Savona, P. B. Littlewood, B. Deveaud, and L. Si Dang, Nature 443, 409 (2006).

${ }^{3}$ A. Kavokin, J. J. Baumberg, G. Malpuech, and F. P. Laussy, Microcavities (Clarendon Press, Oxford, 2006).

${ }^{4}$ H. Deng, H. Haug, and Y. Yamamoto, Rev. Mod. Phys. 82, 1489 (2010).

${ }^{5}$ S. Christopoulos, G. Baldassarri Höger von Högersthal, A. J. D. Grundy, P. G. Lagoudakis, A. V. Kavokin, J. J. Baumberg, G. Christmann, R. Butté, J.-F. Carlin, and N. Grandjean, Phys. Rev. Lett. 98, 126405 (2007).

${ }^{6} \mathrm{~F}$. Li, L. Orosz, O. Kamoun, S. Bouchoule, C. Brimont, P. Disseix, T. Guillet, X. Lafosse, M. Leroux, J. Leymarie, M. Mexis, M. Mahailovic, G. Patriarche, F. Réveret, D. Solnyshkov, J. Zuniga-Perez, and G. Malpuech, Phys. Rev. Lett. 110, 196406 (2013).

${ }^{7}$ S. Kéna-Cohen and S. R. Forrest, Nat. Photonics 4, 371 (2010).

${ }^{8}$ J. D. Plumhof, T. Stöferle, L. Mai, U. Scherf, and R. F. Mahrt, Nat. Mater. 13, 247 (2014).

${ }^{9}$ K. S. Daskalakis, S. A. Maier, R. Murray, and S. Kéna-Cohen, Nat. Mater. 13, 271 (2014).

${ }^{10}$ C. Schneider, A. Rahimi-Iman, N. Y. Kim, J. Fischer, I. G. Savenko, M. Amthor, M. Lermer, A. Wolf, L. Worschech, V. D. Kulakovskii, I. A. Shelykh, M. Kamp, S. Reitzenstein, A. Forchel, Y. Yamamoto, and S. Höfling, Nature 497, 348 (2013).

${ }^{11}$ P. Bhattacharya, B. Xiao, A. Das, S. Bhowmick, and J. Heo, Phys. Rev. Lett. 110, 206403 (2013).

${ }^{12}$ T. Espinosa-Ortega and T. C. H. Liew, Phys. Rev. B 87, 195305 (2013).

${ }^{13}$ T. Gao, P. S. Eldridge, T. C. H. Liew, S. I. Tsintzos, G. Stavrinidis, G. Deligeorgis, Z. Hatzopoulos, and P. G. Savvidis, Phys. Rev. B 85, 235102 (2012). 
${ }^{14}$ D. Ballarini, M. De Giorgi, E. Cancellieri, R. Houdré, E. Giacobino, R. Cingolani, A. Bramati, G. Gigli, and D. Sanvitto, Nat. Commun. 4, 1778 (2013).

${ }^{15}$ C. Sturm, D. Tanese, H. S. Nguyen, H. Flayac, E. Gallopin, A. Lemaitre, I. Sagnes, D. Solnyshkov, A. Amo, G. Malpuech, and J. Bloch, Nat. Commun. 5, 3278 (2014).

${ }^{16}$ D. A. B. Miller, D. S. Chemla, T. C. Damen, A. C. Gossard, W. Wiegmann, T. H. Wood, and C. A. Burrus, Phys. Rev. B 32, 1043 (1985).

${ }^{17}$ J. Gessler, V. Baumann, M. Emmerling, M. Amthor, K. Winkler, S. Höfling, C. Schneider, and M. Kamp, Appl. Phys. Lett. 105, 181107 (2014).

${ }^{18}$ T. A. Fisher, A. M. Afshar, D. M. Whittaker, M. S. Skolnick, J. S. Roberts, G. Hill, and M. A. Pate, Phys. Rev. B 51, 2600 (1995).

${ }^{19}$ P. Tsotsis, S. I. Tsintzos, G. Christmann, P. G. Lagoudakis, O. Kyriienko, I. A. Shelykh, J. J. Baumberg, A. V. Kavokin, Z. Hatzopoulos, P. S. Eldridge, and P. G. Savvidis, Phys. Rev. Appl. 2, 014002 (2014).

${ }^{20}$ T. C. H. Liew, A. V. Kavokin, T. Ostatnický, M. Kaliteevski, I. A. Shelykh, and R. A. Abram, Phys. Rev. B 82, 033302 (2010).
${ }^{21}$ K. Kurihara, T. Numai, I. Ogura, A. Yasuda, M. Sugimoto, and K. Kasahara, J. Appl. Phys. 73, 21 (1993).

${ }^{22}$ J. Erland, V. Mizeikis, W. Langbein, J. R. Jensen, and J. M. Hvam, Phys. Rev. Lett. 86, 5791 (2001).

${ }^{23}$ D. Bajoni, P. Senellart, E. Wertz, I. Sagnes, A. Miard, A. Lemaître, and J. Bloch, Phys. Rev. Lett. 100, 047401 (2008).

${ }^{24}$ J. Shah, R. C. C. Leite, and J. F. Scott, Solid State Commun. 8, 1089 (1970).

${ }^{25}$ M. S. Skolnick, T. A. Fisher, and D. M. Whittaker, Semicond. Sci. Technol. 13, 645 (1998).

${ }^{26}$ E. Vigil and P. Diaz, Cryst. Res. Technol. 19, 285 (1984).

${ }^{27}$ G. Bastard, E. E. Mendez, L. L. Chang, and L. Esaki, Phys. Rev. B 28, 3241 (1983).

${ }^{28}$ H. Flayac and I. Savenko, Appl. Phys. Lett. 103, 201105 (2013).

${ }^{29}$ J. Fischer, I. G. Savenko, M. D. Fraser, S. Holzinger, S. Brodbeck, M. Kamp, I. A. Shelykh, C. Schneider, and S. Höfling, Phys. Rev. Lett. 113, 203902 (2014).

${ }^{30}$ K. Winkler, P. Gold, B. Bradel, S. Reitzenstein, V. D. Kulakovskii, M. Kamp, C. Schneider, and S. Höfling, Phys. Rev. B 91, 045127 (2015). 